\title{
The heterogeneity of antipsychotic response in the treatment of schizophrenia
}

\author{
M. Case ${ }^{1}$, V. L. Stauffer ${ }^{1}$, H. Ascher-Svanum ${ }^{2}$, R. Conley ${ }^{1}$, S. Kapur ${ }^{3}$, J. M. Kane ${ }^{4}$, S. Kollack-Walker ${ }^{1}$, \\ J. Jacob ${ }^{1 *}$ and B. J. Kinon ${ }^{2}$ \\ ${ }^{1}$ Lilly USA, LLC, Indianapolis, IN, USA \\ ${ }^{2}$ Eli Lilly and Company, Indianapolis, IN, USA \\ ${ }^{3}$ Institute of Psychiatry, King's College London, UK \\ ${ }^{4}$ Zucker Hillside Hospital, Glen Oaks, NY, USA
}

\begin{abstract}
Background. Schizophrenia is a heterogeneous disorder in terms of patient response to antipsychotic treatment. Understanding the heterogeneity of treatment response may help to guide treatment decisions. This study was undertaken to capture inherent patterns of response to antipsychotic treatment in patients with schizophrenia, characterize the subgroups of patients with similar courses of response, and examine illness characteristics at baseline as possible predictors of response.
\end{abstract}

Method. Growth mixture modeling (GMM) was applied to data from a randomized, double-blind, 12-week study of 628 patients with schizophrenia or schizo-affective disorder treated with risperidone or olanzapine.

Results. Four distinct response trajectories based on Positive and Negative Syndrome Scale (PANSS) total score over 12 weeks were identified: Class 1 (420 patients, 80.6\%) with moderate average baseline PANSS total score showing gradual symptom improvement; Class 2 (65 patients, 12.5\%) showing rapid symptom improvement; Class 3 (24 patients, $4.6 \%$ ) with high average baseline PANSS total score showing gradual symptom improvement; and Class 4 (12 patients, $2.3 \%$ ) showing unsustained symptom improvement. Latent class membership of early responders (ER) and early non-responders (ENR) was determined based on $20 \%$ symptom improvement criteria at 2 weeks and ultimate responders (UR) and ultimate non-responders (UNR) based on $40 \%$ symptom improvement criteria at 12 weeks. Baseline factors with potential influence on latent class membership were identified.

Conclusions. This study identified four distinct treatment response patterns with predominant representation of responders or non-responders to treatment in these classes. This heterogeneity may represent discrete endophenotypes of response to treatment with different etiologic underpinnings.

Received 22 March 2010; Revised 2 August 2010; Accepted 21 August 2010; First published online 7 October 2010

Key words: Antipsychotic drugs, endophenotypes, growth mixture modeling, heterogeneity, response patterns, schizophrenia.

\section{Introduction}

Schizophrenia is a heterogeneous disorder in terms of response to antipsychotic treatment. Several studies have found that about $70 \%$ of patients fail to experience at least minimal response early in treatment (Kinon et al. 1993, 2008; Ascher-Svanum et al. 2008). Currently available medications for schizophrenia are effective for only about $50 \%$ of patients (Kerwin \& Osborne, 2000; Lieberman et al. 2005; Miyamoto et al. 2005). Poor symptom response is associated with premature treatment discontinuation, symptom

* Address for correspondence: J. Jacob, Ph.D., Lilly Corporate

Center, DC 4133, Indianapolis, IN 46285, USA.

(Email: Jacob_Jayanthi@lilly.com) exacerbations, relapse, and increased risk of hospitalization with resultant higher costs of treatment (Ayuso-Gutierrez \& del Rio Vega, 1997; Perkins, 2002; Thieda et al. 2003; Liu-Seifert et al. 2005). There is, therefore, a need to better understand the characteristics of the group of patients who are non-responsive to treatment and to find better treatment options for them.

There may be two important areas to observe predictors of response: (1) disease state at baseline before initiation of treatment; and (2) early symptom changes after commencement of treatment. Cognitive functioning deficits (Harvey et al. 2005), poor pre-morbid functioning (Rabinowitz et al. 2006), earlier age of onset (Meltzer et al. 1997), duration of untreated psychosis (Ucok et al. 2004; Perkins, 2005) and male

The online version of this article is published within an Open Access environment subject to the conditions of the Creative Commons Attribution-NonCommercial-ShareAlike licence <http://creativecommons.org/licenses/by-nc-sa/2.5/>. The written permission of Cambridge University Press must be obtained for commercial re-use. 
gender (Aleman et al. 2003) are baseline factors that have been found to be associated with poor treatment response. Patients likely to remain as non-responders to treatment based on their early non-response to treatment at weeks 1 or 2 have been reported. Correll et al. (2003) observed that early non-response to treatment, as measured by a $20 \%$ reduction in Brief Psychiatric Rating Scale (BPRS) total score at week 1, predicted non-response at 4 weeks for $100 \%$ of patients. Other studies have suggested the hypothesis that early non-response to treatment within the first 2 weeks of treatment initiation is a good indicator of treatment refractoriness (Correll et al. 2003; Leucht et al. 2007; Kinon et al. 2008).

Estimates of response from early symptom changes have used a priori cut-off scores (percentage improvement) to define treatment response and do not account for inter-individual variation in drug response. Furthermore, the focus has been on early response at a particular time point, which could be 1 or 2 weeks, depending on the study. Embedded trajectories in antipsychotic treatment response without a priori cutoffs for improvement in symptoms or a time point for early response may be able to bring new insight into treatment response patterns to antipsychotic drugs. Growth mixture modeling (GMM) has been explored as an alternative method to classify clinical progression patterns. This is a technique that identifies homogeneous trajectories from varying individual responses. Recent applications of GMM in medical research include trajectories of change in depression severity during treatment with antidepressants (Uher et al. 2010) and examination of placebo response in antidepressant trials (Muthén \& Brown, 2009). The objectives of this analysis were to: (1) capture inherent patterns of response to risperidone or olanzapine treatment in patients with schizophrenia; (2) characterize the subgroups of patients with similar courses of treatment response; and (3) examine various illness characteristics at baseline before initiation of treatment as possible predictors of response.

\section{Method}

\section{Study design}

Data for this study were obtained from a randomized, double-blind, parallel, 12-week study (Kinon et al. 2010) consisting of 628 patients (aged 18-65 years) meeting the diagnostic criteria for schizophrenia, schizo-affective disorder or schizophreniform disorder according to DSM-IV. The trial was conducted in accordance with the Declaration of Helsinki and all applicable regulatory requirements. This study was conducted at 64 study centers in three countries from
May 2006 to December 2007. Eligible patients provided written informed consent before undergoing any study procedure or receiving any study treatment.

Details of the study design consisting of three study periods and inclusion/exclusion criteria can be found in the primary manuscript of this clinical trial (Kinon et al. 2010). Study period I consisted of screening of patients. Study period II consisted of patients receiving 2 weeks of open-label treatment with risperidone 2 to $6 \mathrm{mg} /$ day and, at the end of 2 weeks, patients were classified as early responders (ER) or early nonresponders (ENR) based on a-priori criteria of $20 \%$ improvement in Positive and Negative Syndrome Scale (PANSS; Kay et al. 1987) total scores. Details regarding the $20 \%$ improvement criteria for the PANSS scores are given in the primary manuscript (Kinon et al. 2010). After early study discontinuation, $83 \%$ of patients (522/628) entered study period III doubleblinded (post-baseline data were not available for one patient). Early responders ( $\geqslant 20 \%$ improvement in PANSS total score at week 2) to risperidone treatment continued the same treatment for another 10 weeks. Early non-responders $(<20 \%$ improvement in PANSS total score at week 2) to risperidone treatment were randomly assigned in a 1:1 ratio to either risperidone 2-6 mg/day or olanzapine 10 to $20 \mathrm{mg}$ / day for another 10 weeks of treatment (see Supplementary Fig. 1. available online).

For the current analysis, the hypothesis is that longitudinal response profiles may exist that can better characterize response to treatment compared to cross-sectional dichotomization of response/nonresponse based on a-priori degree of categorical symptom improvement after a specified duration of treatment. Therefore, the analysis was conducted using pooled treatment groups of risperidone and olanzapine.

\section{Statistical analysis}

To investigate the heterogeneity in treatment response, we applied GMM. GMM is a combination of growth models to examine the development of individuals on an outcome variable (PANSS total, PANSS positive, and PANSS negative scores) over time and a mixture model to identify subpopulations, referred to as response patterns/trajectories in this paper, using categorical latent variables. Growth modeling uses random coefficients, termed growth factors, to allow for individual differences in development within response trajectories. GMM uses outcome at all time points, allows for missingness of data under a missing at random assumption, and allows estimation of the probability of an individual's membership to the identified trajectories. GMM was fit into 


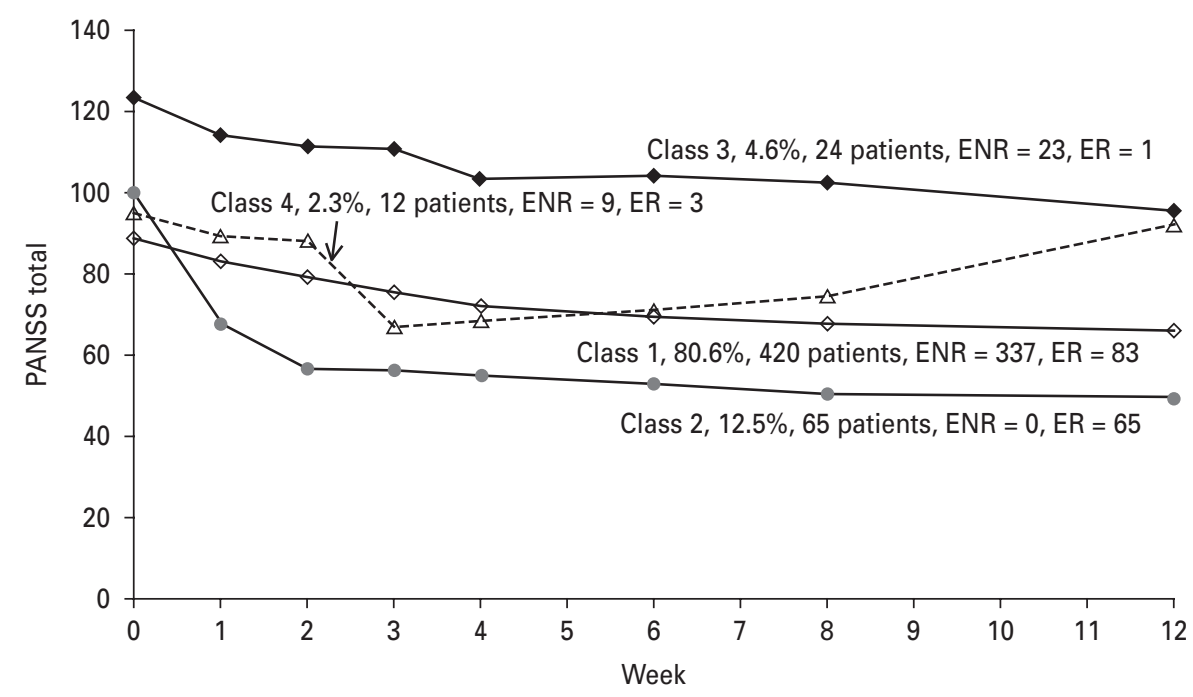

Fig. 1. Growth mixture modeling (GMM) analysis of the Positive and Negative Syndrome Scale (PANSS) total score reveals four distinct latent classes of treatment response. ER, early responders ( $\geqslant 20 \%$ improvement in PANSS total at week 2$)$; ENR, early non-responders.

the general latent variable framework of the Mplus program (Muthén \& Muthén, 2007). The parameters of this model are estimated in the maximum-likelihood framework using an expectation-maximization (EM) algorithm. A broad overview of GMM can be found in the Handbook of Quantitative Methodology for the Social Sciences (Muthén, 2004).

GMM was applied to PANSS total, PANSS positive, and PANSS negative scores at eight time points (visits) over 12 weeks of study. A piece-wise GMM model was used to model trajectories. Baseline and the first three post-baseline visits until week 3 were modeled quadratically (linear and quadratic growth factor) and the remaining four visits over 9 weeks were modeled linearly (linear growth factors). Conceptually, an individual's PANSS total score and subscores can be understood by identifying the trajectory they belong to and then applying the trajectory-specific growth factors. Throughout this paper, estimated mean growth factors are presented. To identify the appropriate number of response trajectories, the Bayesian Information Criterion (BIC) was used. To test for differences between identified trajectories (classes), analysis of variance (ANOVA) was used for continuous variables and Fisher's exact test was used for categorical variables, with class as the independent variable.

\section{Measures}

The distribution of ER, ENR, ultimate responders (UR: $\geqslant 40 \%$ improvement in PANSS total score at endpoint) and ultimate non-responders (UNR: $<40 \%$ improvement in PANSS total score at endpoint) in the identified latent classes was determined.
Secondary efficacy measures studied included the Montgomery-Asberg Depression Rating Scale (MADRS; Montgomery \& Asberg, 1979), the Clinical Global Impression (CGI) Severity and Improvement scale (Guy, 1976), and the Brief Assessment of Cognition in Schizophrenia (BACS; Keefe et al. 2004). Quality of life measures included the Heinrich Carpenter Quality of Life Scale (QLS; Heinrichs et al. 1984), the Subjective Well-being Under Neuroleptics (SWN) scale (Naber et al. 2001) the and Schizophrenia Objective Functioning Instrument (SOFI; Kleinman et al. 2009). Extrapyramidal symptoms (EPS) were measured by the Abnormal Involuntary Movement Scale (AIMS; Guy, 1976), the Barnes Akathisia Scale (BAS; Barnes, 1989) and the Modified Simpson-Angus Rating Scale (SA; Simpson \& Angus, 1970).

\section{Results}

GMM analysis of PANSS total scores for all patients treated with risperidone or olanzapine over 12 weeks revealed four distinct response patterns as shown in Fig. 1 and described below. The four-class model was selected from five different sequential piece-wise models using BIC, with a smaller BIC indicating a better model (see Supplementary Table 1, available online).

\section{Class 1: Gradual symptom improvement and moderate average baseline PANSS score (420 patients, $80.6 \%$ )}

The average baseline PANSS total score for patients in this class was 88.7 points with a subsequent average 

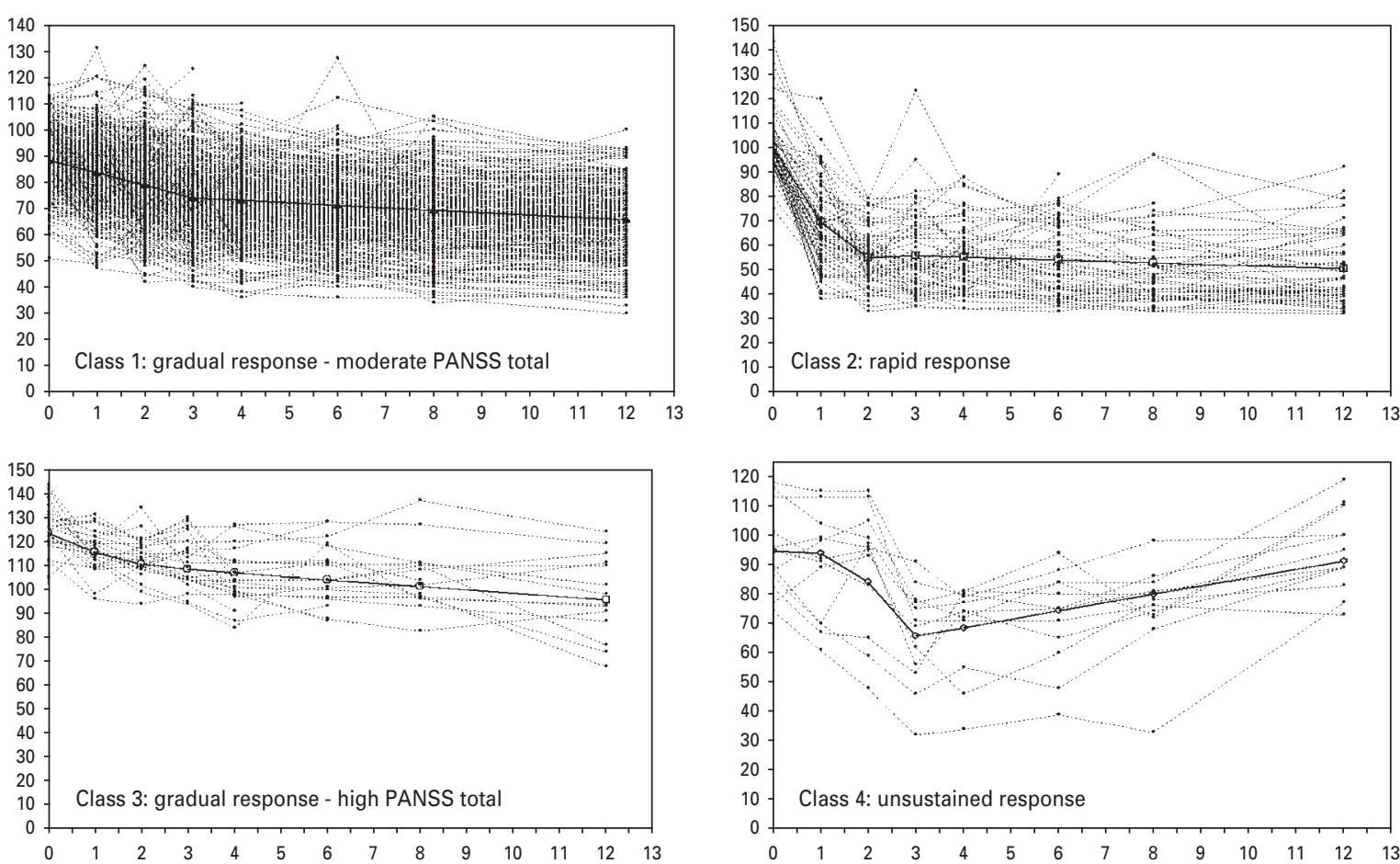

Fig. 2. The observed trajectories of individuals classified into the four latent classes are shown as broken lines and the solid line represents the model-estimated means shown in Fig. 1. PANSS, Positive and Negative Syndrome Scale.

improvement of 22.5 points after 12 weeks of treatment. Patients in this class were a mixture of responders and non-responders to treatment with mostly non-responders, consisting of $80 \%$ ENR and $87 \%$ UNR patients; $\geqslant 89 \%$ of ENR patients continued to UNR status.

The responding patients in this class may be characterized as gradual responders to treatment, with response distributed throughout the 12-week treatment period. Fifty-five patients in this class reached ultimate response $(13.1 \%)$ and constituted $10.6 \%$ of the total patients.

\section{Class 2: Rapid symptom improvement (65 patients, $12.5 \%$ )}

The average baseline PANSS total score for patients in this class was 100.1 followed by a subsequent average improvement of 50.9 points after 12 weeks of treatment. Class 2 was uniquely distributed with $100 \%$ ER patients; $66 \%$ progressed to UR after 12 weeks of treatment.

Patients in this class were distinctly rapid responders to treatment, with rapid initial response for the first 2 weeks, followed by a more gradual response for the next 10 weeks. Forty-three patients in this class reached ultimate response $(66 \%)$ and constituted $8.3 \%$ of the total patients.
Class 3: Gradual symptom improvement and high average baseline PANSS score (24 patients, $4.6 \%$ )

The average baseline PANSS total score for patients in this class was 123.7 points with a subsequent average improvement of 28.0 points after 12 weeks of treatment. The trajectory shape of this class was similar to Class 1, but with a higher average PANSS total score at baseline compared to Class 1 . Class 3 was uniquely distributed with $96 \%$ ENR and $96 \%$ UNR patients; $\geqslant 96 \%$ ENR patients continued to UNR status.

\section{Class 4: Unsustained symptom improvement (12 patients, $2.3 \%$ )}

The average baseline PANSS total score for patients in this class was 95.1, showing initial average symptom improvement of 27.9 points followed by worsening of symptoms. Class 4 was represented by $75 \%$ ENR and $100 \%$ UNR patients; all of the ENR patients continued to UNR status.

The observed trajectories of individuals classified into the four latent classes are shown in Fig. 2. The patient demographics for the four latent classes are shown in Table 1 . The four classes were significantly different in age at onset, ethnicity, EPS, PANSS total, CGI severity, MADRS, QLS total, SOFI global, and 
Table 1. Demographics at baseline for patients in the four latent classes of PANSS total score GMM analysis

\begin{tabular}{|c|c|c|c|c|c|}
\hline & $\begin{array}{l}\text { Class } 1 \\
\text { (420 patients) }\end{array}$ & $\begin{array}{l}\text { Class } 2 \\
\text { (65 patients) }\end{array}$ & $\begin{array}{l}\text { Class } 3 \\
\text { (24 patients) }\end{array}$ & $\begin{array}{l}\text { Class } 4 \\
\text { (12 patients) }\end{array}$ & $p$ value \\
\hline Gender, male & 62 & 55 & 75 & 50 & 0.302 \\
\hline Age, years & $42.1 \pm 11.0$ & $40.7 \pm 10.4$ & $38.9 \pm 12.4$ & $45.0 \pm 13.3$ & 0.307 \\
\hline Age at onset, years & $25.3 \pm 9.9$ & $26.4 \pm 9.9$ & $20.4 \pm 6.4$ & $30.3 \pm 11.2$ & 0.020 \\
\hline Ethnicity/race & & & & & 0.039 \\
\hline Caucasian & 42.8 & 52.3 & 50 & 66.7 & \\
\hline African descent & 47.9 & 36.9 & 25 & 16.7 & \\
\hline Hispanic & 8.1 & 9.2 & 20.8 & 16.7 & \\
\hline Other & 1.2 & 1.5 & 4.2 & - & \\
\hline Diagnosis & & & & & 0.982 \\
\hline Schizophrenia & 75.2 & 73.8 & 79.2 & 75 & \\
\hline Schizo-affective disorder & 19.0 & 18.5 & 20.8 & 16.7 & \\
\hline Schizophreniform & 5.7 & 7.7 & - & 8.3 & \\
\hline Duration of current episode, days & $27 \pm 80$ & $20 \pm 42$ & $30 \pm 96$ & $21 \pm 37$ & 0.899 \\
\hline Number of previous episodes & $5 \pm 26$ & $3 \pm 3$ & $7 \pm 21$ & $4 \pm 5$ & 0.853 \\
\hline \multicolumn{6}{|l|}{ Extrapyramidal symptoms } \\
\hline AIMS & $0.7 \pm 2.2$ & $0.7 \pm 1.9$ & $4.7 \pm 5.5$ & $1.7 \pm 5.2$ & $<0.001$ \\
\hline BAS total score & $0.5 \pm 1.6$ & $0.6 \pm 1.5$ & $2.2 \pm 2.7$ & $0.2 \pm 0.4$ & $<0.001$ \\
\hline SA total score & $0.9 \pm 2.2$ & $1.2 \pm 2.9$ & $4.9 \pm 5.9$ & $1.5 \pm 4.3$ & $<0.001$ \\
\hline BACS score & $41.0 \pm 22.0$ & $36.8 \pm 18.9$ & $37.7 \pm 31.4$ & $41.3 \pm 35.0$ & 0.514 \\
\hline PANSS total score & $88.6 \pm 10.6$ & $100.7 \pm 11.2$ & $125.1 \pm 9.1$ & $94.6 \pm 14.9$ & $<0.001$ \\
\hline CGI severity score & $4.5 \pm 0.6$ & $5.0 \pm 0.5$ & $5.0 \pm 0.7$ & $4.8 \pm 0.6$ & $<0.001$ \\
\hline CGI improvement score & $4.1 \pm 0.5$ & $4.2 \pm 0.6$ & $4.0 \pm 0.6$ & $4.1 \pm 0.7$ & 0.206 \\
\hline MADRS total score & $15.5 \pm 8.8$ & $17.5 \pm 9.1$ & $23.3 \pm 8.0$ & $23.9 \pm 10.2$ & $<0.001$ \\
\hline Quality of Life Scale total score & $52.8 \pm 21.0$ & $48.3 \pm 20.8$ & $32.6 \pm 15.8$ & $47.6 \pm 21.5$ & $<0.001$ \\
\hline SOFI global score & $57.8 \pm 16.8$ & $52.3 \pm 15.3$ & $40.1 \pm 12.2$ & $49.0 \pm 15.2$ & $<0.001$ \\
\hline SWN total score & $78.2 \pm 16.8$ & $70.0 \pm 16.3$ & $71.1 \pm 18.0$ & $72.3 \pm 14.0$ & $<0.001$ \\
\hline Body mass index, $\mathrm{kg} / \mathrm{m}^{2}$ & $30.6 \pm 8.2$ & $30.9 \pm 9.4$ & $27.9 \pm 5.7$ & $28.2 \pm 8.9$ & 0.306 \\
\hline
\end{tabular}

AIMS, Abnormal Involuntary Movement Scale; BACS, Brief Assessment of Cognition in Schizophrenia; BAS, Barnes Akathisia Scale; CGI, Clinical Global Impression; GMM, growth mixture modeling; MADRS, Montgomery-Asberg Depression Rating Scale; PANSS, Positive and Negative Syndrome Scale; SA, Modified Simpson-Angus Scale; SOFI, Schizophrenia Objective Functioning Instrument; SWN, Subjective Well-being under Neuroleptics.

Values given as percentage or mean \pm standard deviation.

SWN total scores and were primarily driven by differences between group 3 and the remaining groups.

Because Class 1 was a combination of UR and UNR patients, these subgroups were separated and the baseline demographics compared in Table 2 ; the major differences were a lower number of previous illness episodes and a higher age at onset for UR patients compared to UNR patients.

Baseline demographics of UNR patients from Class 1 are compared with UNR patients from Class 3 in Table 2. UNR patients in these two classes were significantly different in age at onset, ethnicity, EPS, PANSS total, CGI severity, MADRS, QLS total, SOFI global, and SWN total scores Baseline demographics of gradual responders (UR from Class 1) are compared with rapid responders (UR from Class 2) in Table 2. Gradual and rapid responders were significantly different in PANSS total, CGI severity, and SWN total scores. However, the $p$ values in Tables 1 and 2 should be interpreted as hypothesis generating as opposed to hypothesis confirming.

GMM analysis of PANSS negative scores also revealed four classes similar to the PANSS total analysis. Analysis of PANSS positive scores did not indicate Class 4 (unsustained response) as observed in the PANSS total and PANSS negative analysis. Class membership of ER/ENR and UR/UNR patients in the PANSS negative and PANSS positive scores analysis was similar to the PANSS total scores analysis (data not included).

\section{Comparison of PANSS total score GMM analysis of the total patient group versus the early non-responder group}

PANSS total score GMM analysis of 370 ENR patients was compared with the GMM analysis of the total 
Table 2. Comparison of baseline patient demographics for UNR in Class 3, UNR in Class 1, UR in Class 1 (gradual responders), and UR in Class 2 (rapid responders) from the PANSS total score analysis

\begin{tabular}{|c|c|c|c|c|c|c|}
\hline & $\begin{array}{l}\text { UNR Class } 3 \\
(n=23)\end{array}$ & $\begin{array}{l}\text { UNR Class } 1 \\
(n=365)\end{array}$ & $p$ value & $\begin{array}{l}\text { Gradual } \\
\text { responders } \\
\text { UR Class } 1 \\
(n=55)\end{array}$ & $\begin{array}{l}\text { Rapid } \\
\text { responders } \\
\text { UR Class } 2 \\
(n=43)\end{array}$ & $p$ value \\
\hline Gender, male & 73.9 & 62.7 & 0.373 & 58.2 & 62.8 & 0.682 \\
\hline Age, years & $39.2 \pm 12.7$ & $42.2 \pm 11.0$ & 0.199 & $41.4 \pm 11.3$ & $39.8 \pm 11.2$ & 0.484 \\
\hline Age at onset, years & $20.4 \pm 6.6$ & $24.8 \pm 9.6$ & 0.031 & $28.4 \pm 11.4$ & $25.2 \pm 8.8$ & 0.135 \\
\hline Ethnicity/race & & & 0.021 & & & 0.517 \\
\hline Caucasian & 47.8 & 43.0 & & 41.8 & 55.8 & \\
\hline African descent & 26.1 & 48.5 & & 43.6 & 30.2 & \\
\hline Hispanic & 21.7 & 7.4 & & 12.7 & 11.6 & \\
\hline Other & 4.3 & 1.1 & & 1.8 & 2.3 & \\
\hline Diagnosis & & & 1.000 & & & 0.627 \\
\hline Schizophrenia & 78.3 & 74.5 & & 80.0 & 74.4 & \\
\hline Schizo-affective & 21.7 & 19.7 & & 14.5 & 18.6 & \\
\hline Schizophreniform & - & 5.8 & & 5.5 & 7.0 & \\
\hline Duration of current episode, days & $30 \pm 96$ & $27 \pm 85$ & 0.868 & $25 \pm 40$ & $22 \pm 50$ & 0.754 \\
\hline Number of previous episodes & $7 \pm 21$ & $6 \pm 27$ & 0.802 & $3 \pm 2$ & $3 \pm 4$ & 0.553 \\
\hline \multicolumn{7}{|l|}{ Extrapyramidal symptoms } \\
\hline AIMS & $4.9 \pm 5.6$ & $0.6 \pm 1.9$ & $<0.001$ & $1.6 \pm 3.4$ & $0.5 \pm 1.6$ & 0.058 \\
\hline BAS total score & $2.3 \pm 2.7$ & $0.5 \pm 1.5$ & $<0.001$ & $0.8 \pm 1.9$ & $0.3 \pm 1.0$ & 0.055 \\
\hline SA total score & $5.1 \pm 6.0$ & $0.8 \pm 2.0$ & $<0.001$ & $1.7 \pm 3.4$ & $1.0 \pm 2.8$ & 0.330 \\
\hline BACS score & $37.6 \pm 32.1$ & $41.3 \pm 21.5$ & 0.431 & $38.9 \pm 25.2$ & $34.7 \pm 16.6$ & 0.349 \\
\hline PANSS total scores & $125.2 \pm 9.3$ & $88.0 \pm 10.6$ & $<0.001$ & $92.5 \pm 10.0$ & $103.0 \pm 10.9$ & $<0.001$ \\
\hline CGI improvement score & $4.0 \pm 0.5$ & $4.1 \pm 0.5$ & 0.387 & $4.2 \pm 0.6$ & $4.2 \pm 0.4$ & 0.863 \\
\hline CGI severity score & $5.0 \pm 0.7$ & $4.5 \pm 0.6$ & $<0.001$ & $4.8 \pm 0.6$ & $5.1 \pm 0.3$ & 0.004 \\
\hline MADRS total score & $23.5 \pm 8.1$ & $15.7 \pm 8.8$ & $<0.001$ & $14.1 \pm 8.7$ & $17.6 \pm 8.3$ & 0.051 \\
\hline Quality of Life Scale total score & $33.3 \pm 15.7$ & $52.7 \pm 20.7$ & $<0.001$ & $53.6 \pm 23.6$ & $45.0 \pm 19.3$ & 0.058 \\
\hline SOFI global score & $40.3 \pm 12.5$ & $58.1 \pm 16.7$ & $<0.001$ & $55.7 \pm 18.0$ & $49.3 \pm 15.0$ & 0.067 \\
\hline SWN total score & $70.7 \pm 18.3$ & $78.2 \pm 16.5$ & 0.044 & $78.5 \pm 18.6$ & $67.3 \pm 13.9$ & 0.002 \\
\hline Body mass index, $\mathrm{kg} / \mathrm{m}^{2}$ & $27.5 \pm 5.4$ & $30.6 \pm 8.2$ & 0.072 & $30.7 \pm 7.8$ & $28.8 \pm 7.7$ & 0.218 \\
\hline
\end{tabular}

AIMS, Abnormal Involuntary Movement Scale; BACS, Brief Assessment of Cognition in Schizophrenia; BAS, Barnes Akathisia Scale; CGI, Clinical Global Impression; GMM, growth mixture modeling; MADRS, Montgomery-Asberg Depression Rating Scale; PANSS, Positive and Negative Syndrome Scale; SA, Modified Simpson-Angus Scale; SOFI, Schizophrenia Objective Functioning Instrument; SWN, Subjective Well-being under Neuroleptics; UR, Ultimate responder ( $\geqslant 40 \%$ improvement in PANSS total at 12 weeks); UNR, ultimate non-responder.

Values given as percentage or mean \pm standard deviation.

patient group. Three overlapping classes with similar distributions of patients were observed. Classes 1, 2 and 3 in the ENR group consisted of 22, 8 and 339 patients respectively, and the all-patient group consisted of 23, 9 and 337 patients respectively.

\section{Discussion}

The GMM analysis of symptom progression and response among patients with schizophrenia treated with risperidone or olanzapine for 12 weeks revealed heterogeneity in treatment response to antipsychotic treatment. The analysis provided an insight into the complexity of antipsychotic response across individuals and identified four distinct trajectories (classes) of response. The majority of patients were distributed in a class characterized by gradual improvement in illness symptoms (Class 1: 80.6\%, 365/ 420 patients were UNR). The next major trajectory was characterized by rapid improvement in symptoms for the first 2 weeks, followed by a more gradual improvement over the next 10 weeks of treatment (Class 2: $12.5 \%, 43 / 65$ patients were UR). Classes 3 and 4 were smaller, with Class $3(4.6 \%)$ having the highest baseline PANSS total score and gradual symptom improvement, and Class $4(2.3 \%)$ characterized by an unsustained improvement in illness symptoms; both Classes 3 and 4 consisted of $\geqslant 96 \%$ UNR patients. 


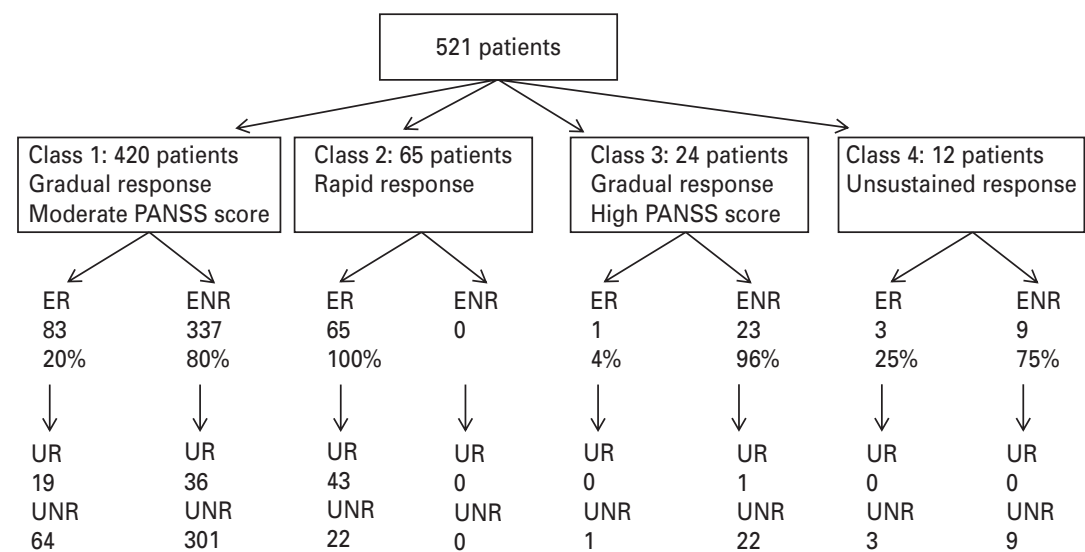

Fig. 3. Treatment response characteristics of patients in the four classes. ER, early responders ( $\geqslant 20 \%$ improvement in PANSS total at week 2); ENR, early non-responders; UR, ultimate responders ( $\geqslant 40 \%$ improvement in PANSS total at 12 weeks); UNR, ultimate non-responders; PANSS, Positive and Negative Syndrome Scale.

Treatment response characteristics of patients in the four classes are summarized in Fig. 3.

\section{Responders to treatment in Class 2}

Class 2 was uniquely represented by $100 \%$ of ER patients. The subgroup of rapid responders to treatment has been identified in several studies. Marques et al. (in press) identified a subset of rapid responders in a placebo-controlled study in which 420 patients with schizophrenia were treated for 6 weeks with haloperidol or olanzapine. The rapid responders were exclusively from the drug-treated group and showed $>70 \%$ improvement and consisted of $17 \%$ of the drug-treated group. Levine \& Rabinowitz (2010) also identified a subgroup of rapid responders in their study of 497 patients with first-episode schizophrenia randomly assigned to risperidone or haloperidol. The rapid responders showed 59\% improvement in PANSS total score during the first 4 weeks of treatment and consisted of $19 \%$ of the treatment group. A subgroup of rapid responders termed as early persistent responders was identified in data from three 6-week, double-blind, placebo-controlled trials in patients with schizophrenia (Glick et al. 2009). A rapid decrease in mean PANSS total scores of 40.7 points was observed in $23.5 \%$ of patients. Unlike the results from Marques et al. (in press), rapid response was also observed in the placebo-treated group (14.2\%; mean decrease in PANSS total: 37.3 points). A subgroup of rapid responders was also identified in a depression study consisting of 807 patients treated with escitalopram or nortriptyline for 12 weeks (Uher et al. 2010). The majority of patients (75\%) were described as gradual responders whereas a smaller group $(25 \%)$ displayed rapid response for the first 3 weeks followed by a gradual response over the remaining treatment period.
Rapid response to treatment in patients with schizophrenia has been associated with baseline excess production of dopamine as measured by elevated levels of the dopamine metabolite homovanillic acid in plasma (pHVA; Garver et al. 2000). Prior to initiation of treatment with haloperidol, patients $(n=32)$ were assessed for $\mathrm{pHVA}$ levels. Patients with high $\mathrm{pHVA}$ displayed a $>30 \%$ reduction in BPRS psychosis score by day 4 after initiating treatment. A delayed-response psychosis was shown to be associated with low-tonormal pHVA. A significant relationship has been reported between dopamine $\mathrm{D}_{2}$ receptor (DRD2) genetic variation and treatment response in patients with firstepisode schizophrenia (Lencz et al. 2006).

The rapid responders in this study (Class 2) were found to be more ill than responders in Class 1, yet displayed dramatic illness improvement. As it is well accepted that schizophrenia may have complex genetic underpinnings, the subset of rapid responders repeatedly observed in schizophrenia studies may represent a unique endophenotype that is responsive to antipsychotic treatment. Endophenotypes are characteristics reflecting actions of fewer genes than the more complex phenotype of schizophrenia. Identifying endophenotypes in schizophrenia may aid in understanding the disease pathology and in developing new and effective medications. Additional research focusing on specific genetic testing may lend support to this idea.

\section{Rapid and gradual responders to treatment in Classes 1 and 2}

Responders to antipsychotic treatment were distributed mainly in Classes 1 and 2. Patients in Class 2 were rapid responders whereas those in Class 1 were gradual responders. The literature has been divided about early onset and delayed onset of response to 
treatment. The delayed onset hypothesis proposes that response to treatment occurs after about 2-3 weeks of antipsychotic treatment (Agid et al. 2003). The early onset hypothesis proposes more improvement in the earlier weeks than in the later weeks of antipsychotic treatment. It is possible that the delayed onset and early onset hypotheses reflect the gradual and rapid responders respectively. A similar observation has been reported in a study consisting of 1128 patients receiving paliperidone extended-release or placebo for 6 weeks (Glick et al. 2009). An early persistent response (rapid response) was observed in $23.5 \%$ of patients and a late persistent response (gradual response) in $16.3 \%$ of patients in the drug-treated group.

\section{Non-responders to treatment in Classes 3 and 4}

Ultimate non-responders to treatment were distributed in all classes, with the majority being distributed in Class $1(70 \%)$. UNR patients in Classes 3 and 4 (combined total: $6.7 \%$ ) were unique in that they represented a class consisting of almost all $(\geqslant 96 \%)$ UNR patients and were characterized by a greater severity of depressive symptoms as assessed by the MADRS. Non-responding patients in Classes 3 and 4 may belong to an endophenotype representing treatment-refractory patients.

\section{Baseline predictors of response to antipsychotic treatment}

The baseline characteristics of UNR patients from Class $3(4.4 \%)$ were distinctly different from UNR patients from Class $1(70 \%)$. UNR patients from Class 3 were characterized with high extrapyramidal (AIMS, BAS and SA) and depression (MADRS) symptoms, low quality of life measures (SOFI, SWN and QLS), younger age at onset of illness, Hispanic ethnicity, and higher CGI severity and PANSS total scores (all with significant $p$ values). Younger age at onset of illness (Meltzer et al. 1997), high EPS (Rabinowitz et al. 2006), poor social functioning (Haro et al. 2008) and depressive symptoms (Moller, 2005) have been associated with poor treatment response. These baseline characteristics of Class 3 patients were also observed in the GMM analysis of PANSS positive and PANSS negative scores analysis (data not included). These results indicate a strikingly worse clinical and functional profile at baseline for one subgroup of UNR patients (Class 3). Although the UNR patients in the two classes (Classes 1 and 3) did not attain the a-priori criteria for response, how these two groups of patients might compare with longer duration of treatment or with a change in treatment regimen is worth further study, in addition to understanding what seems to be a greater preponderance of patients with a Hispanic origin in patients with the worst clinical status.

UR patients (gradual responders in Class 1 and rapid responders in Class 2) were observed to have a lower number of previous episodes compared to UNR patients in Classes 1 and 3. A higher number of previous episodes as a predictor of poor treatment outcome has been reported in a cluster analysis of 1449 patients with schizophrenia (Lipkovich et al. 2009). Rapid responders were differentiated from gradual responders by a higher psychopathology scores (CGI severity and PANSS total) and a lower SWN score (all significant $p$ values).

\section{Accuracy of $20 \%$ symptom improvement at 2 weeks to classify early non-responders to treatment}

Early non-responders to treatment were distributed in Classes 1, 3 and 4 . The accuracy of the a priori $20 \%$ symptom improvement cut-off at week 2 to classify ENR patients is indicated by the high percentage of ENR patients at week 2 continuing to UNR status at week 12 in these classes. Comparison of the GMM analysis of the total patient group versus the 370 ENR group revealed three overlapping classes. Therefore, the $20 \%$ a-priori improvement cut-off at 2 weeks to identify non-responding patients early in the treatment may be a useful and accurate tool. However, there is a greater risk of misclassifying a responder to treatment as ENR at 2 weeks in Class 1 (gradual responder) compared to Classes 3 and 4 .

\section{Limitations}

The four-class solution to these data is a function of the sample size and the study population. With larger sample sizes, it is possible that other classes may emerge or the current large classes may split into subclasses. Additional research focusing on specific genetic testing may lend support to the rapid responders and treatment-refractory non-responder endophenotypes. This analysis consisted of patients in a clinical trial setting; in the naturalistic patient population with polypharmacy, the observed trajectories may change.

\section{Conclusions}

This study identified four distinct treatment response patterns in patients with schizophrenia treated with risperidone or olanzapine. The majority of patients were distributed in a class characterized by gradual improvement in illness symptoms (Class 1: 80.6\%). A unique class distributed predominantly with ER 
patients showing a rapid improvement in illness symptoms for the first 2 weeks followed by a more gradual improvement may represent a subset of patients responsive to atypical antipsychotic drugs (Class 2). Among responders to treatment, two types of response were observed: gradual responders and rapid responders. Classes 3 and 4 consisting of almost all UNR patients may represent treatment-refractory patients. This heterogeneity may represent discrete endophenotypes of response to treatment with different etiologic underpinnings. Current findings also show that the a-priori definition of early non-response to treatment seems to be an accurate threshold and a useful tool for predicting longer symptom response and trajectory class. Baseline PANSS total score, EPS, depressive symptoms, quality of life measures, and age at onset may have potential influence on membership in the latent classes.

\section{Note}

Supplementary material accompanies this paper on the Journal's website (http://journals.cambridge.org/ psm).

\section{Declaration of Interest}

M. Case, V. L. Stauffer, H. Ascher-Svanum, R. Conley, S. Kollack-Walker, J. Jacob and B. J. Kinon are all employees of Eli Lilly and Company. Dr J. Kane serves as a consultant and on speakers' bureaus for the following commercial organizations: AstraZeneca (speaker), Bristol-Myers Squibb (speaker), Cephalon, Dainippon Sumitomo, GlaxoSmithKline, Intracellullar Therapeutics, Janssen (speaker), Johnson \& Johnson, Eli Lilly and Company (speaker), Otsuka America Pharmaceutical, Inc. (speaker), Pfizer, PGxHealth, Proteus, Takeda, Vanda, and Wyeth. Dr S. Kapur has had financial association with the following commercial organizations in the past three years: grant support from AstraZeneca, Bristol-Myers Squibb, and GlaxoSmithKline; consulting/scientific advisor/ speaking engagements with AstraZeneca, Bioline, Bristol-Myers Squibb, Eli Lilly, Janssen (Johnson \& Johnson), Lundbeck, Otsuka, Organon, Pfizer, Servier, and Solay Wyeth.

\section{Acknowledgements}

This study was funded by Eli Lilly and Company. We thank H. Fox, Medical Information Specialist with Eli Lilly and Company for overseeing the editing this manuscript. We also thank R. Conway and C. Modeas, both of i3 Statprobe, for editorial assistance. i3 Statprobe is a division of Ingenix, which is a subsidiary of United Health Group, with whom Eli Lilly contracted the editing and formatting of this manuscript.

\section{References}

Agid O, Kapur S, Arenovich T, Zipursky RB (2003). Delayed-onset hypothesis of antipsychotic action: a hypothesis tested and rejected. Archives of General Psychiatry 60, 1228-1235.

Aleman A, Kahn RS, Selten JP (2003). Sex differences in the risk of schizophrenia: evidence from meta-analysis. Archives of General Psychiatry 60, 565-571.

Ascher-Svanum H, Nyhius AW, Faris DE, Kinon BJ, Baker RW, Shekar A (2008). Clinical, functional, and economic ramifications of early nonresponse to antipsychotics in the naturalistic treatment of schizophrenia. Schizophrenia Bulletin 34, 1163-1171.

Ayuso-Gutierrez JL, del Rio Vega JM (1997). Factors influencing relapse in the long-term course of schizophrenia. Schizophrenia Research 28, 199-206.

Barnes TR (1989). A rating scale for drug-induced akathisia. British Journal of Psychiatry 154, 672-676.

Correll CU, Malhotra AK, Kaushik S, McMeniman M, Kane JM (2003). Early prediction of antipsychotic response in schizophrenia. American Journal of Psychiatry 160, 2063-2065.

Garver DL, Holcomb JA, Christensen JD (2000). Heterogeneity of response to antipsychotics from multiple disorders in the schizophrenia spectrum. Journal of Clinical Psychiatry 61, 964-972.

Glick ID, Bossie CA, Alphs L, Canuso CM (2009). Onset and persistence of antipsychotic response in patients with schizophrenia. Journal of Clinical Psychopharmacology 29, 542-547.

Guy W (1976). ECDEU Assessment Manual for Psychopharmacology, Revised. Publication ADM 76-338. US Department of Health, Education, and Welfare: Rockville, MD.

Haro JM, Novick D, Suarez D, Ochoa S, Roca M (2008). Predictors of the course of illness in outpatients with schizophrenia: a prospective three year study. Progress in Neuro-Psychopharmacology and Biological Psychiatry 32, 1287-1292.

Harvey PD, Rabinowitz J, Eerdekens M, Davidson M (2005). Treatment of cognitive impairment in early psychosis: a comparison of risperidone and haloperidol in a large long-term trial. American Journal of Psychiatry 162, 1888-1895.

Heinrichs DW, Hanlon TE, Carpenter Jr. WT (1984). The Quality of Life Scale: an instrument for rating the schizophrenic deficit syndrome. Schizophrenia Bulletin 10, 388-398.

Kay SR, Fiszbein A, Opler LA (1987). The positive and negative syndrome scale (PANSS) for schizophrenia. Schizophrenia Bulletin 13, 261-276.

Keefe RSE, Goldberg TE, Harvey PD, Gold JM, Poe MP, Coughenour L (2004). The Brief Assessment of Cognition in Schizophrenia: reliability, sensitivity, and comparison 
with a standard neurocognitive battery. Schizophrenia Research 68, 283-297.

Kerwin RW, Osborne S (2000). Antipsychotic drugs. Medicine 28, 23-25.

Kinon BJ, Chen L, Ascher-Svanum H, Stauffer VL, Kollack-Walker S, Sniadecki JL, Kane JM (2008). Predicting response to atypical antipsychotics based on early response in the treatment of schizophrenia. Schizophrenia Research 102, 230-240.

Kinon BJ, Chen L, Ascher-Svanum H, Stauffer VL, Kollack-Walker S, Zhou W, Kapur S, Kane JM (2010). Early response to antipsychotic drug therapy as a clinical marker of subsequent response in the treatment of schizophrenia. Neuropsychopharmacology 35, 581-590.

Kinon BJ, Kane JM, Johns C, Perovich R, Ismi M, Koreen A, Weiden P (1993). Treatment of neuroleptic-resistant schizophrenic relapse. Psychopharmacology Bulletin 29, 309-314.

Kleinman L, Lieberman J, Dube S, Mohs R, Zhao Y, Kinon B, Carpenter W, Harvey PD, Green MF, Keefe RS, Frank L, Bowman L, Revicki DA (2009). Development and psychometric performance of the schizophrenia objective functioning instrument: an interviewer administered measure of function. Schizophrenia Research 107, 275-285.

Lencz T, Robinson DG, Xu K, Ekholm J, Sevy S, Gunduz-Bruce H, Woerner MG, Kane JM, Goldman D, Malhotra AK (2006). DRD2 promoter region variation as a predictor of sustained response to antipsychotic medication in first-episode schizophrenia patients. American Journal of Psychiatry 163, 529-531.

Leucht S, Busch R, Kissling W, Kane JM (2007). Early prediction of antipsychotic nonresponse among patients with schizophrenia. Journal of Clinical Psychiatry 68 352-360.

Levine SZ, Rabinowitz J (2010). Trajectories and antecedents of treatment response over time in early-episode psychosis. Schizophrenia Bulletin 36, 624-632.

Lieberman JA, Stroup TS, McEvoy JP, Swartz MS, Rosenheck RA, Perkins DO, Keefe RS, Davis SM, Davis CE, Lebowitz BD, Severe J, Hsiao JK; Clinical Antipsychotic Trials of Intervention Effectiveness (CATIE) Investigators (2005). Effectiveness of antipsychotic drugs in patients with chronic schizophrenia. New England Journal of Medicine 353, 1209-1223.

Lipkovich I, Deberdt W, Csernansky JG, Buckley P, Peuskens J, Kollack-Walker S, Rotelli M, Houston JP (2009). Defining 'good' and 'poor' outcomes in patients with schizophrenia or schizoaffective disorder: a multidimensional data-driven approach. Psychiatry Research 170, 161-167.

Liu-Seifert H, Adams D, Kinon BJ (2005). Discontinuation of treatment of schizophrenic patients is driven by poor symptom response: a pooled post-hoc analysis of four atypical antipsychotic drugs. BMC Medicine 3, 21.

Marques TR, Arenovich T, Agid O, Sajeev G, Muthén B, Chen LC, Kinon BJ, Kapur S (in press). The different trajectories of antipsychotic response: antipsychotics versus placebo. Psychological Medicine.
Meltzer HY, Rabinowitz J, Lee MA, Cola PA, Ranjan R, Findling RL, Thompson PA (1997). Age at onset and gender of schizophrenic patients in relation to neuroleptic resistance. American Journal of Psychiatry 154, 475-482.

Miyamoto S, Duncan GE, Marx CE, Lieberman JA (2005). Treatments for schizophrenia: a critical review of pharmacology and mechanisms of action of antipsychotic drugs. Molecular Psychiatry 10, 79-104.

Moller HJ (2005). Occurrence and treatment of depressive comorbidity/cosyndromality in schizophrenic psychoses: conceptual and treatment issues. World Journal of Biological Psychiatry 6, 247-263.

Montgomery SA, Asberg M (1979). A new depression scale designed to be sensitive to change. British Journal of Psychiatry 134, 382-389.

Muthén B (2004). Latent variable analysis: growth mixture modeling and related techniques for longitudinal data. In Handbook of Quantitative Methodology for the Social Sciences (ed. D. Kaplan), pp. 345-368. Sage Publications: Newbury Park, CA.

Muthén B, Brown H (2009). Estimating drug effects in the presence of placebo response: causal inference using growth mixture modeling. Statistics in Medicine 28, 3363-3385.

Muthén B, Muthén L (2007). Mplus: Statistical Analysis with Latent Variables: User's Guide, 5th edn. Muthén and Muthén: Los Angeles, CA.

Naber D, Moritz S, Lambert M, Rajonk FG, Holzbach R, Mass R, Andresen B (2001). Improvement of schizophrenic patients' subjective well-being under atypical antipsychotic drugs. Schizophrenia Research 50, 79-88.

Perkins DO (2002). Predictors of noncompliance in patients with schizophrenia. Journal of Clinical Psychiatry 63, 1121-1128.

Perkins DO (2005). Relationship between duration of untreated psychosis and outcome in first-episode schizophrenia : a critical review and meta-analysis. American Journal of Psychiatry 162, 1785-1804.

Rabinowitz J, Harvey PD, Eerdekens M, Davidson M (2006). Premorbid functioning and treatment response in recent-onset schizophrenia. British Journal of Psychiatry 189, 31-35.

Simpson GM, Angus JW (1970). A rating scale for extrapyramidal side effects. Acta Psychiatrica Scandinavica (Suppl.) 212, 11-19.

Thieda P, Beard S, Richter A, Kane J (2003). An economic review of compliance with medication therapy in the treatment of schizophrenia. Psychiatric Services 54, 508-516.

Ucok A, Polat A, Genc A, Cakir S, Tarun N (2004). Duration of untreated psychosis may predict acute treatment response in first-episode schizophrenia. Journal of Psychiatric Research 38, 163-168.

Uher R, Muthén B, Souery D, Mors O, Jaracz J, Placentino A, Petrovic A, Zobel A, Henigsberg N, Rietschel M, Aitchison KJ, Farmer A, McGuffin P (2010). Trajectories of change in depression severity during treatment with antidepressants. Psychological Medicine 40, 1367-1377. 